\begin{tabular}{|c|c|}
\hline Title & Precise slit-width control of niobium apertures for superconducting LEDs \\
\hline Author(s) & $\begin{array}{l}\text { Huh, Jae Hoon; Hermannstädter, Claus; Sato, Hiroyasu; Ito, Saki; Idutsu, Y asuhiro; Sasakura, Hirotaka; Tanaka, } \\
\text { Kazunori; A kazaki, Tatsushi; Suemune, Ikuo }\end{array}$ \\
\hline Citation & $\begin{array}{l}\text { Nanotechnology, 22(4), 045302 } \\
\text { https://doi.org/10.1088/0957-4484/22/4/045302 }\end{array}$ \\
\hline Issue Date & 2011-01-28 \\
\hline Doc URL & http://hdl .handle.net/2115/44767 \\
\hline Rights & $\begin{array}{l}\text { This is an author-created, un-copyedited version of an article accepted for publication in Nanotechnology. IOP } \\
\text { Publishing Ltd is not responsible for any errors or omissions in this version of the manuscript or any version derived } \\
\text { from it. The definitive publisher authenticated version is avail able online at } 10.1088 / 0957-4484 / 22 / 4 / 045302\end{array}$ \\
\hline Type & article (author version) \\
\hline File Information & Nan22-4_045302.pdf \\
\hline
\end{tabular}

Instructions for use 


\title{
Precise slit-width control of niobium apertures for superconducting LEDs
}

\author{
Jae-Hoon Huh ${ }^{1,2}$, Claus Hermannstädter ${ }^{1}$, Hiroyasu Sato ${ }^{1}$, Saki Ito ${ }^{1}$, Yasuhiro \\ Idutsu $^{1}$, Hirotaka Sasakura ${ }^{1,2}$, Kazunori Tanaka ${ }^{2,3}$, Tatsushi Akazaki ${ }^{2,4}$, \\ and Ikuo Suemune ${ }^{1,2}$
}

\author{
${ }^{1}$ Research Institute for Electronic Science, Hokkaido University, Sapporo 001-0021, Japan \\ ${ }^{2}$ Japan Science and Technology Corporation(CREST), 4-1-8 Honcho Kawaguchi, Saitama 332-0012, Japan \\ ${ }^{3}$ Central Laboratory, Hamamatsu Photonics, Hiraguchi, Hamamatsu, Shizuoka 434-8601, Japan \\ ${ }^{4}$ NTT Basic Research Laboratory, Wakamiya, Morinosato, Atsugi, kanagawa, 243-0198, Japan \\ E-mail:hjaehoon@es.hokudai.ac.jp
}

\begin{abstract}
We introduce a novel three step procedure for precise niobium (Nb)-etching on the nm-scale, including the design of high contrast resist patterning and sacrifice layer formation under high radio frequency (RF) power. We present the results of precise slit fabrication using this technique and discuss its application for the production of superconducting devices, such as, superconductor-semiconductor-superconductor (S-Sm-S) Josephson junctions. For the reactive ion etching (RIE) of $\mathrm{Nb}$ we selected $\mathrm{CF}_{4}$ as etchant gas and a positive tone resist to form the etching mask. We found that the combination of resist usage and RIE process allows for etching of thicker Nb layers when utilizing the opposite dependence of the etching rate (ER) on the $\mathrm{CF}_{4}$ pressure in the case of $\mathrm{Nb}$ as compared to the resist. Precise slit-width control of 80 nm-thick and 200 nm-thick Nb apertures was performed with three kinds of ER control, for the resist, the $\mathrm{Nb}$, and the underlying layer. S-Sm-S Josephson junctions were fabricated with lengths as small as $80 \mathrm{~nm}$, which can be considered clean and short and thus exhibit critical currents as high as $50 \mu \mathrm{A}$. Moreover, possible further applications as for apertures of superconducting light emitting diodes (SC LEDs) are addressed.
\end{abstract}

\section{Introduction}

On-demand generation of single photons and entangled photon pairs in the telecommunication band is not possible with the common state-of-the-art conventional single photon sources such as using down conversion [1]. These types of sources produce Poissonian distributed photon pairs with a high photon number fluctuation, which requires a strong attenuation in order to suppress multi-photon events $(\mathrm{n} \geq 2)$ and, consequently, makes the sources quite inefficient. Therefore, solid-state photon sources generating on-demand single photons and entangled photon pairs are highly desired for quantum information processing and communication (figure $1(\mathrm{a}, \mathrm{b})$ ). We have proposed a new scheme to realize solid-state entangled photon pair sources using a SC LED [2-5], as highlighted in figure 1(c). Spontaneous recombination of electron cooper pairs penetrating from the $\mathrm{Nb}$ cathode and holes from the anode lead to the generation of entangled 
photon pairs [5]. The emission of entangled photon pairs can be observed through the aperture of a SC Nb cathode. Figure 2 describes in detail that the penetration of Cooper pairs into the semiconductor and the emission efficiency depends on the size and depth of the aperture and on the thickness of the SC electrodes. For wider apertures the photon extraction efficiency increases, whereas for narrower apertures the Cooper pair injection is enhanced. Recently, K. Il'in et al. [6] reported that a decrease of the thickness is accompanied by a decrease of both the critical temperature $T_{c}$ and the critical current $I_{c}$, which demonstrates that the deposition of a thicker SC Nb layer provides a stronger proximity effect.

Over the past two decades, RIE of Nb has been employed to fabricate functional SC materials with attractive properties with a high SC $T_{c}$ and thermal cycling capability; e.g., for Josephson tunnel junctions, SC quantum interference devices (SQUIDs), signal processors, optical switching devices, and emission extraction apertures of SC LEDs [5-10]. Mixed gases like $\mathrm{CF}_{4} / \mathrm{O}_{2}, \mathrm{SF}_{6} / \mathrm{O}_{2}, \mathrm{CCl}_{2} \mathrm{~F}_{2} / \mathrm{O}_{2}, \mathrm{Cl}_{2} / \mathrm{CHCl}_{3}, \mathrm{CCl}_{2} \mathrm{~F}_{2} / \mathrm{H}_{2}$ and single gases like $\mathrm{CF}_{4}, \mathrm{CCl}_{2} \mathrm{~F}_{2}$ and $\mathrm{CBrF}_{3}$ have been reported as gas etchant of $\mathrm{Nb}$ [10-14]. $\mathrm{CF}_{4}$ was selected in our work, considering not only its selectivity of $\mathrm{Nb}$ etching but also the gas resistance of the resist and underlying InGaAs layer. In this work, we report that the combination of resist and RIE processes, without the usage of a metal or $\mathrm{SiO}_{2}$ mask, enables us to achieve $200 \mathrm{~nm}$-deep $\mathrm{Nb}$ etching. This deep etching is possible because of the opposite $\mathrm{CF}_{4}$ pressure dependence of the $\mathrm{Nb}$ and the resist ERs.

For the nanofabrication of SC apertures, the development of high-resolution electron-beam lithography (EBL) is important. We have successfully achieved high contrast patterning which is consistent with computer aided design (CAD) structures and sizes. It is crucial to have precise control over the aperture size and shape to improve both the emission efficiency and the smooth Cooper pair injection into the underlying active layer by proximity effect. In this regard, the formation of different aperture sizes and shapes is investigated. Precise slit-width control of the $200 \mathrm{~nm}$-thick Nb apertures can be obtained by using isotropic etching after the formation of side-wall sacrifice layers based on high contrast resist patterning. S-Sm-S Josephson junctions with narrow Sm-slits have been studied and high values of $I_{c}$ were measured that confirm the successful injection of Cooper pairs up to temperatures close to $T_{c}$.

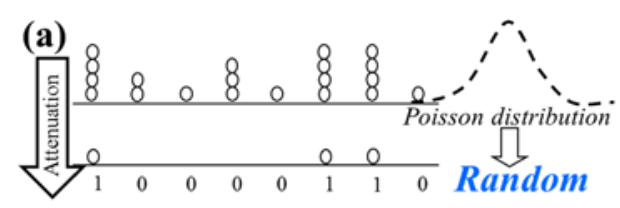

(b)
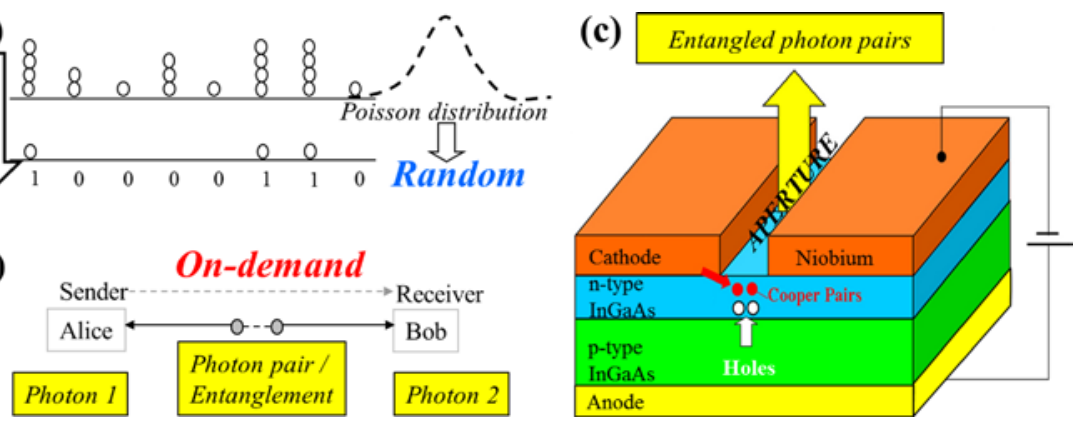

Figure 1: Single photon sources for highly secure quantum communication; (a) generation of single photons using an attenuated source, and (b) on-demand (deterministic) generation of entangled photon pairs for quantum communication. (c) Schematic illustration of the fabricated SC LED proposed for entangled photon-pair generation resulting from radiative recombination of electron Cooper pairs with normal holes. 


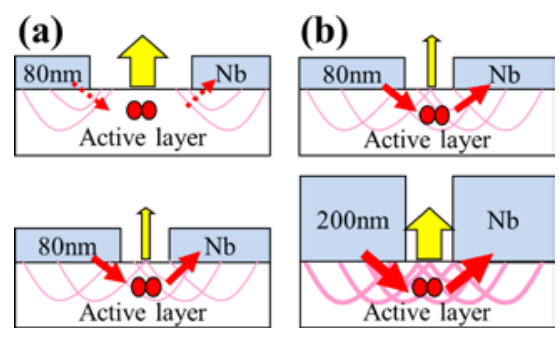

Figure 2: Relationship between extraction efficiency and proximity effect. (a) Slit-width dependence of Nb apertures, where the top image illustrates high photon extraction and the lower one more efficient Cooper pair penetration to the aperture center. (b) SC layer thickness dependence of Nb apertures where the lower image illustrates more efficient Cooper pair injection.

\section{Fabrication and experimental procedure}

The Nb films (80 and $200 \mathrm{~nm}$ thick $\mathrm{Nb}$ ) were deposited by EB evaporation on GaAs substrates. The resist mask for the $\mathrm{Nb}$ etching must allow high EB resolution. The positive tone ZEP 520A resist (ZEP in the following) has been selected because of its high dry etching resistance. The EBL process employing the ZEP is as follows: the spin coating of the resist $(45 \mathrm{~s}, 4000 \mathrm{rpm})$ on the $\mathrm{Nb} / \mathrm{GaAs}$ substrate $(10 \mathrm{~mm} \times 10 \mathrm{~mm})$ was followed by a one day drying time in the vacuum chamber. The coated resist thickness (typically around 440 $\mathrm{nm}$ ) was measured using a Filmetrics F20 thin-film analyzer. The designed line and space patterns (10 - 600 $\mathrm{nm}$ ) were generated by ultra-high- precision EBL (ELS-7700H, ELIONIX) using $100 \mathrm{keV}$ accelerating voltage, $1.8 \mathrm{~nm}$ minimum electron probe diameter and $50 \mathrm{pA}$ beam current. The resist was developed with ZED-N50 at $-17{ }^{\circ} \mathrm{C}$, under immersed state in a bath temperature controller in ethylene glycol solvent diluted with water (1:1 ratio). The low temperature condition was chosen to minimize the undercut and to ensure the precise transfer of the CAD specified structure. The developing solution temperature was accurately measured and kept constant throughout the developing process. The long developing time of 10 min was necessary to provide sufficient dissolution due to the reduced sensitivity at low temperature.

A SAMCO Model 10-NR RIE etcher was used for etching of the Nb apertures. High-resolution fabrication of an $80 \mathrm{~nm}$-thick $\mathrm{Nb}$ electrode was achieved by RIE-CF 4 plasma etching under the following conditions: chamber pressure $=5 \mathrm{~Pa}$, RF power $=100 \mathrm{~W}$, and flow rate $=89 \mathrm{sccm}$. However, after $4.5 \mathrm{~min}$ etching, the positive-tone ZEP resist was completely removed which only allows etching a maximum of 80 $\mathrm{nm}$ of $\mathrm{Nb}$. Consequently, it was required to increase the chamber pressure or RF power to achieve etching depths larger than $80 \mathrm{~nm}$. For etching of a $200 \mathrm{~nm}$-thick $\mathrm{Nb}$ electrode the following conditions were applied: chamber pressure $=30 \mathrm{~Pa}$, RF power $=200 \mathrm{~W}$, and flow rate $=89 \mathrm{sccm}$; a detailed explanation and the respective results are discussed below in section 3 .

A field-emission scanning electron microscope (SEM) equipped with dimension measurement tool (JEOL JSM-6700FT) was used to estimate the patterned slit-widths using top view images as well as cross-sectional views of the developed resist patterns and $\mathrm{Nb}$ apertures (figures 3, 5, 6 and 8). The structure of the sacrifice layers deposited on both sides of the $\mathrm{Nb}$ sidewall (or Nb surface) after removing the resist was obtained using a digital instruments Nanoscope IIIa atomic force microscope (AFM) (figure 6). 

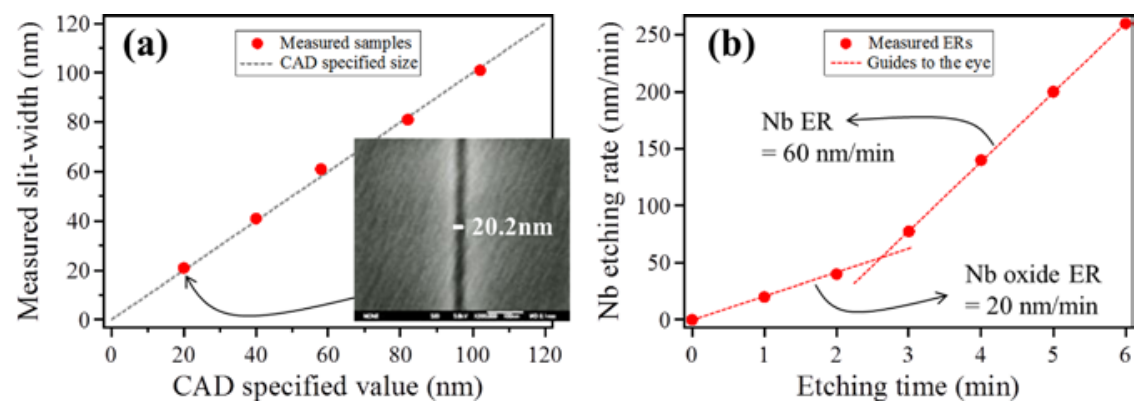

Figure 3: (a) Slit-width control of $80 \mathrm{~nm}$-thick $\mathrm{Nb}$ electrodes, measurement data vs. CAD specified value; the inset shows an SEM image of a successfully fabricated $20.2 \mathrm{~nm}$ Nb slit. (b) Influence of the $\mathrm{Nb}$ oxide on the ERs in the etching process, the $\mathrm{Nb} E R$ is $60 \mathrm{~nm} / \mathrm{min}$, the oxide ER is $20 \mathrm{~nm} / \mathrm{min}$ (etchant $=\mathrm{RIE}_{-} \mathrm{CF}_{4}$, chamber pressure $=5 \mathrm{~Pa}$, flow rate $=89 \mathrm{sccm}, \mathrm{RF}$ power $=100 \mathrm{~W}$, etching time $=3 \mathrm{~min} 20 \mathrm{sec}$ ).

\section{Experimental results and discussion}

Figure 3 shows the $\mathrm{Nb}$ slit-widths after etching of the $80 \mathrm{~nm}$-thick electrodes and the influence of $\mathrm{Nb}$ oxide on the dependency between ER and time. Under the following condition, the etching of the 80 $\mathrm{nm}$-thick $\mathrm{Nb}$ electrodes was very precise with ignorable size variation as compared to the CAD specification: etchant gas $=\mathrm{CF}_{4}$, chamber pressure $=5 \mathrm{~Pa}$, RF power $=100 \mathrm{~W}$, and flow rate $=89 \mathrm{sccm}$ (figure 3(a)). This demonstrates that the combination of low pressure (5 Pa) and low RF power (100 W) etching, based on high contrast resist patterning, can be used for fine patterning. The $20 \mathrm{~nm}$ slit-width aperture without line edge fluctuation was observed by SEM top-view image (inset of figure 3(a)). $80 \mathrm{~nm}$-deep Nb etching can be achieved under the aforementioned etching conditions for etching times between three and four minutes with a remaining thickness of the resist of about $100 \mathrm{~nm}$, where the slower etching of $\mathrm{Nb}$ oxide is considered before the start of $\mathrm{Nb}$ metal etching. Three kinds of oxides $\left(\mathrm{Nb}_{2} \mathrm{O}_{5} / \mathrm{NbO}_{2} / \mathrm{NbO}\right)$ can be generated under natural environment condition during $\mathrm{Nb}$ evaporation, resist coating and its development process [15-18]. Considering the ER of $\mathrm{Nb}$ oxide (20 nm/min) and $\mathrm{Nb}(60 \mathrm{~nm} / \mathrm{min})$ which are highlighted in figure 3(b), the oxide thickness could be estimated to be around $40 \mathrm{~nm}$. However, after 4.5 min etching, the ZEP is completely removed which only allows to etch a maximum of $80 \mathrm{~nm}$ of Nb. As shown in figure 4(a) the 440 $( \pm 20) \mathrm{nm}$ resist thickness for the undiluted case is the thickest achievable layer in a one-time coating process. Following this limitation, it is required to increase the chamber pressure of RIE-CF 4 etching to etch down to depths larger than $80 \mathrm{~nm}$.

We explain the chamber $\mathrm{CF}_{4}$ pressure dependence of the ERs for ZEP, Nb, and (In)GaAs in figure 4(b). At the lowest pressure of $5 \mathrm{~Pa}$, the ZEP ER $(\sim 100 \mathrm{~nm} / \mathrm{min})$ is 5 times higher than that of $\mathrm{Nb}(\sim 20 \mathrm{~nm} / \mathrm{min})$, but the ER ratio of ZEP and $\mathrm{Nb}$ approaches unity $(\sim 35 \mathrm{~nm} / \mathrm{min})$ at the higher pressure of $30 \mathrm{~Pa}$. This is because the higher $\mathrm{CF}_{4}$ pressure enhances the chemical reactivity aspect within the more diffusive regime. The underlying (In)GaAs layer, however, is almost not reactive with $\mathrm{CF}_{4}$ gas. This enables $\mathrm{Nb}$ selective etching for an underlying (In)GaAs active layer, e.g., for SC LED application. 

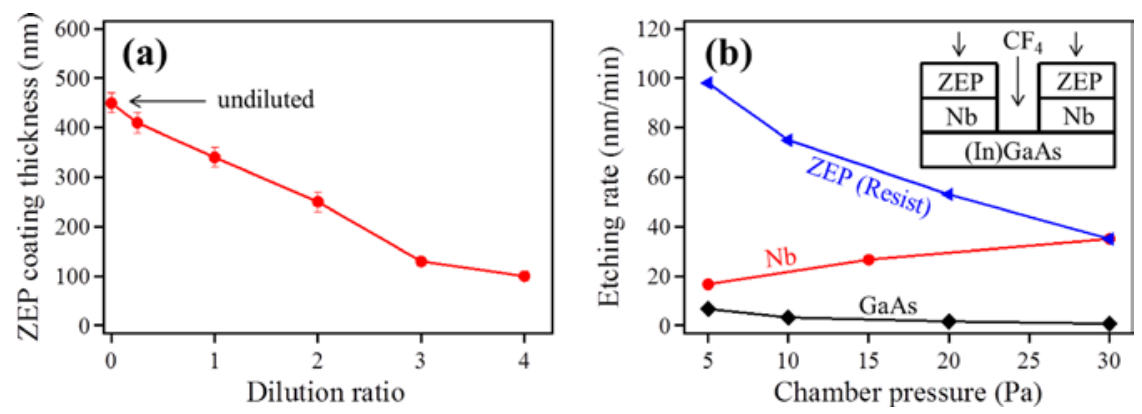

Figure 4: (a) Dependence of the resist coating thickness on the anisole dilution ratio (Dilution ratio = anisole volume/ZEP volume); (b) dependence between the $\mathrm{CF}_{4}$-chamber pressure and the ERs of ZEP resist, $\mathrm{Nb}$ and (In)GaAs.

(a)

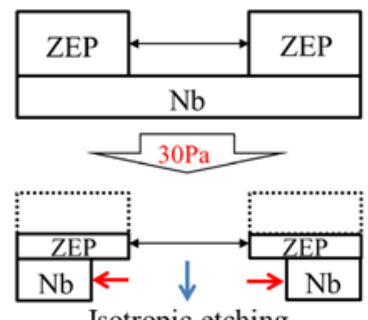

Isotropic etching

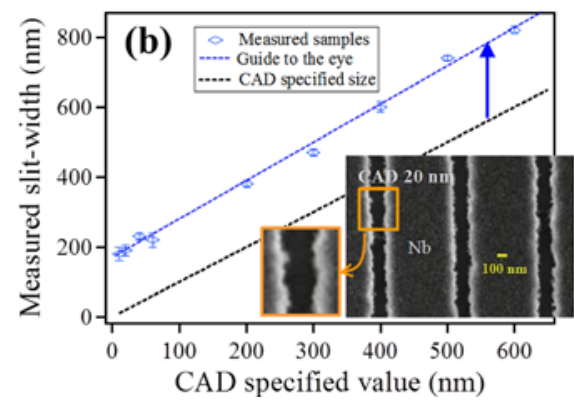

(c)

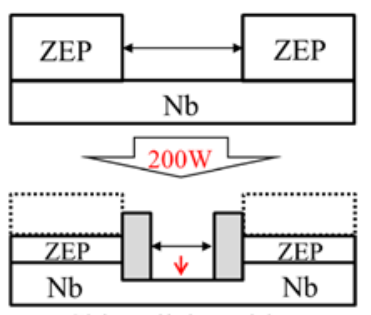

Sidewall deposition

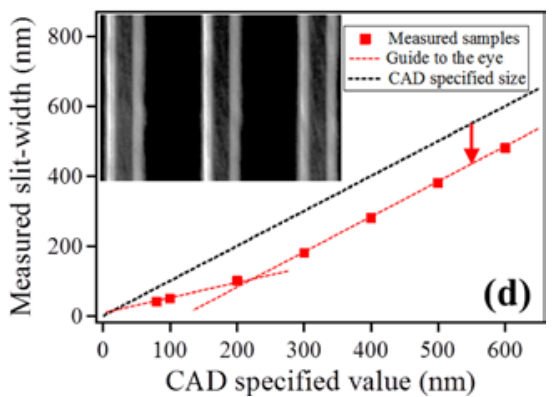

Figure 5: (a) Schematic of the isotropic chemical gas etching reaction under high chamber pressure, and (b) measurement data of the $\mathrm{Nb}$ slit-width after resist removal vs. the CAD specified value. The blue arrow indicates the increased width of the fabricated slits as a result of the side etching. The following conditions were applied: etchant = RIE-CF 4 , chamber pressure $=30 \mathrm{~Pa}$, RF power $=100 \mathrm{~W}$, flow rate $=89 \mathrm{sccm}$, etching time $=4 \mathrm{~min}$. The inset shows an SEM image of an $\mathrm{Nb}$ slit etched under these conditions. (c) Schematic of the sidewall deposition due to the strong ion bombardment under high RF power, and (d) measurement data of the Nb slit-width after resist removal vs. the CAD specified value. The red arrow indicates the reduction of the fabricated slit-width which corresponds to the total thickness of the two deposited side layers. The following conditions were applied: etchant $=$ RIE-CF 4 , chamber pressure $=5 \mathrm{~Pa}, \mathrm{RF}$ power $=200 \mathrm{~W}$, flow rate $=89 \mathrm{sccm}$, etching time $=2 \mathrm{~min}$. The inset shows an SEM image of an Nb slit etched under these conditions.

However, we also found that a higher gas pressure (30 Pa) leads to size expansion of the Nb slit-width because of the strong isotropic chemical reaction which facilitates etchings along both vertical and horizontal directions (figure 5(a)). This isotropic etching can be even more pronounced in wider slits than in the small nano-sized slits because the wider slits provide easier access for the etchant (figure 5(b)). The horizontal 
directional ER was estimated to be between 40 and $65 \mathrm{~nm} / \mathrm{min}$ for 100 - $600 \mathrm{~nm}$ designed slit-widths. Therefore, under high pressure conditions slit-width expansion of 200 - $300 \mathrm{~nm}$ compared to the designed dimensions is unavoidable for apertures in $200 \mathrm{~nm}$ thick Nb electrodes. From the SEM image of figure 5(b), we furthermore observed large line edge roughness which might appear due to an enlargement of the resist pattern fluctuation during the chemical reaction.

We found that a higher RF power $(200 \mathrm{~W}$ ) resulted in edge deposition through a strong ion bombardment. We consider that this phenomenon takes place because the vertical directional physical etching rate is enhanced for higher RF power. This strong physical power can promote the creation of a deposition layer at both sidewalls because the compounds of $\mathrm{Nb}$ as well as the reacted gas adsorbed on the sidewall are stable and cannot easily be etched off (figure 5(c)) [19]. The horizontal widths of each deposition layer accumulated to an almost constant width of around $50 \mathrm{~nm}$ for slits wider than $200 \mathrm{~nm}$ and around a quarter of the silt-width for narrower slits. Furthermore, looking at the SEM image of figure 5(d) we also observed that the deposition layer is higher than the $\mathrm{Nb}$ surface. The compounds of $\mathrm{Nb}$ and reacted gas are accumulated besides the vertical surfaces of both $\mathrm{Nb}$ and ZEP. The structure of the deposition layers remains regardless of the ZEP dissolving reaction during the development process. We propose that these sidewall deposition layers can be used as sacrificial layers against the slit-width expansion due to isotropic etching. Then, the deposition layers can be subsequently removed after the aperture fabrication. A three step processes was established as depicted in figure 6, including the design of high contrast resist patterning, the sacrifice layer formation under high RF power, and the achievement of precise slit-widths by isotropic etching time control.

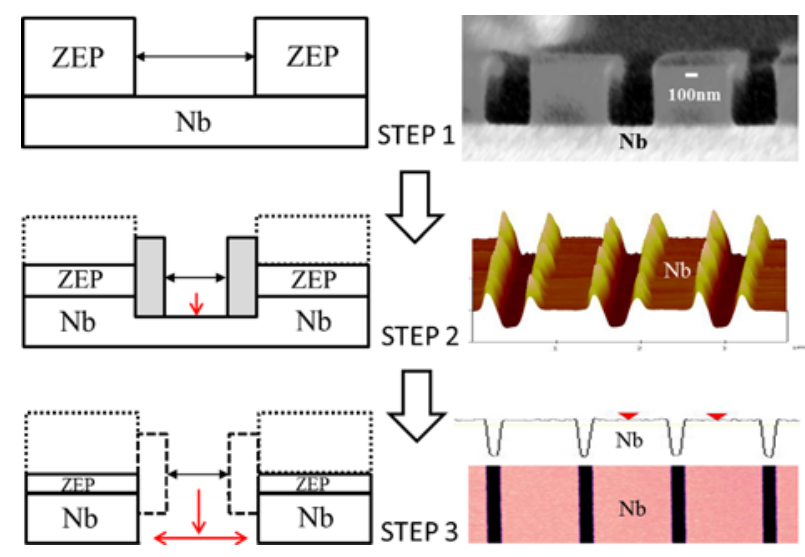

Figure 6: Illustration of the three step mechanism of precise slit-width control for aperture fabrication of $200 \mathrm{~nm}$-thick $\mathrm{Nb}$ electrodes. Schematic (left panel) of the RIE etching mechanism and the corresponding experimental images (right panel: SEM, AFM: cross-sectional and top view with deep profile). Step 1: resist patterning, Step 2: sidewall deposition during high RF-power etching, and Step 3: isotropic etching. (The SEM images for Steps 2 and 3 are given as inset in Fig. 5 (d) and in Fig. 8 (a), respectively.) 


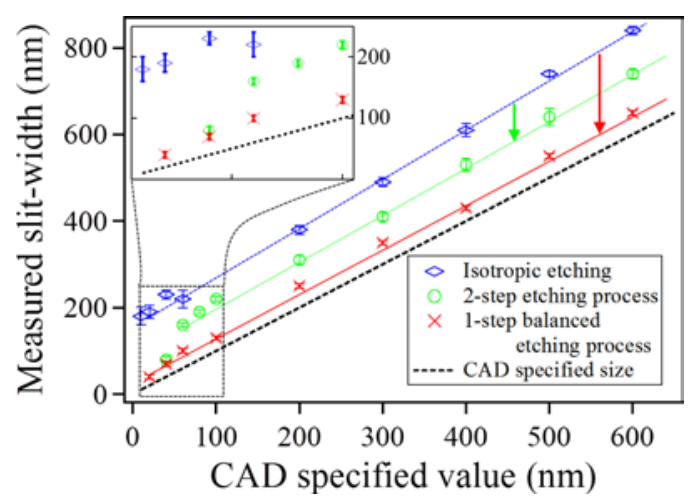

Figure 7: Fabricated Nb slit-width vs. CAD specified value; the results of the 2-step process combining the two unique etching phenomena anisotropic etching and sidewall deposition (green circles) and of the 1-step balancing of these two phenomena (red x) are displayed. The green and red arrows indicate the achieved reduction of the fabricated slit-widths compared to the result of the isotropic etching process (blue diamonds). The inset shows an enlargement of the small size range indicated by the dashed box in order to illustrate the errors, i.e., the slit roughness, more clearly.

Figure 7 shows the results of the aperture fabrication. The improvement is the result of the combination of the two unique phenomena: sidewall etching due to isotropic etching and sidewall deposition (figure 7 , green circles). The combination of sacrifice layer deposition and subsequent isotropic etching reduces the slit-width expansion to around $100 \mathrm{~nm}$ as compared to the pure isotropic etching condition. This effect is a result of a balanced formation and removal of the sidewall sacrifice layers because the reduced slit-widths are consistent with the sacrifice layer widths. It is worthwhile noting that the one-time balanced condition of sidewall deposition and etching (figure 7, red $\mathrm{x}$ ) results in an improved precision to control the aperture slit-widths. It allows the fabrication of apertures as small as $40 \mathrm{~nm}$ and sidewalls of lower roughness, which can be seen in the reduced magnitude of the errors in the respective measurement data (figure 7, red $\mathrm{x}$ error bars).

The SEM image of an $\mathrm{Nb}$ aperture inside the SC LED device (figure 8(a)) highlights the benefit of the described process. It demonstrates how selective etching of an $\mathrm{Nb}$ aperture can be performed without causing damage to the underlying InGaAs active layer because of the opposite dependence of the etching rate on the $\mathrm{CF}_{4}$ pressure for $\mathrm{Nb}$ and $\mathrm{GaAs} / \mathrm{ZEP}$. The precise control of narrow $\mathrm{Nb}$ slit sizes and the accurate and constant slit-width with a low roughness are important properties to fabricate superconducting devices of high performance. To demonstrate the high quality of the nm-sized $\mathrm{Nb}$ etching we studied an Nb-InGaAs-Nb Josephson junction of $L=80 \mathrm{~nm}$ length and $w=20 \mu \mathrm{m}$ width according to the structural design depicted in figures 1(c) and 8(a). Using the low temperature electron mobility of $10^{3} \mathrm{~cm}^{2} \mathrm{~V}^{-1} \mathrm{~s}^{-1}$ and the carrier concentration of $5 \times 10^{18} \mathrm{~cm}^{-3}$ [20] the mean free path $l$ and the thermal coherence length $\xi_{\mathrm{N}}$ in the n-InGaAs layers are estimated as $76 \mathrm{~nm}$ and $400 \mathrm{~nm}$. With these values the Josephson junction under investigation can be considered as (almost) clean and short ( $1 \sim \mathrm{L}<\xi_{\mathrm{N}}$ ). It is worthwhile mentioning that the active layer structure consists of $100 \mathrm{~nm}$ thick n-doped and $100 \mathrm{~nm}$ thick p-doped InGaAs. The transport in the Josephson junction is limited to the n-doped InGaAs because of the built-in potential of the p-n-diode. 
(a)
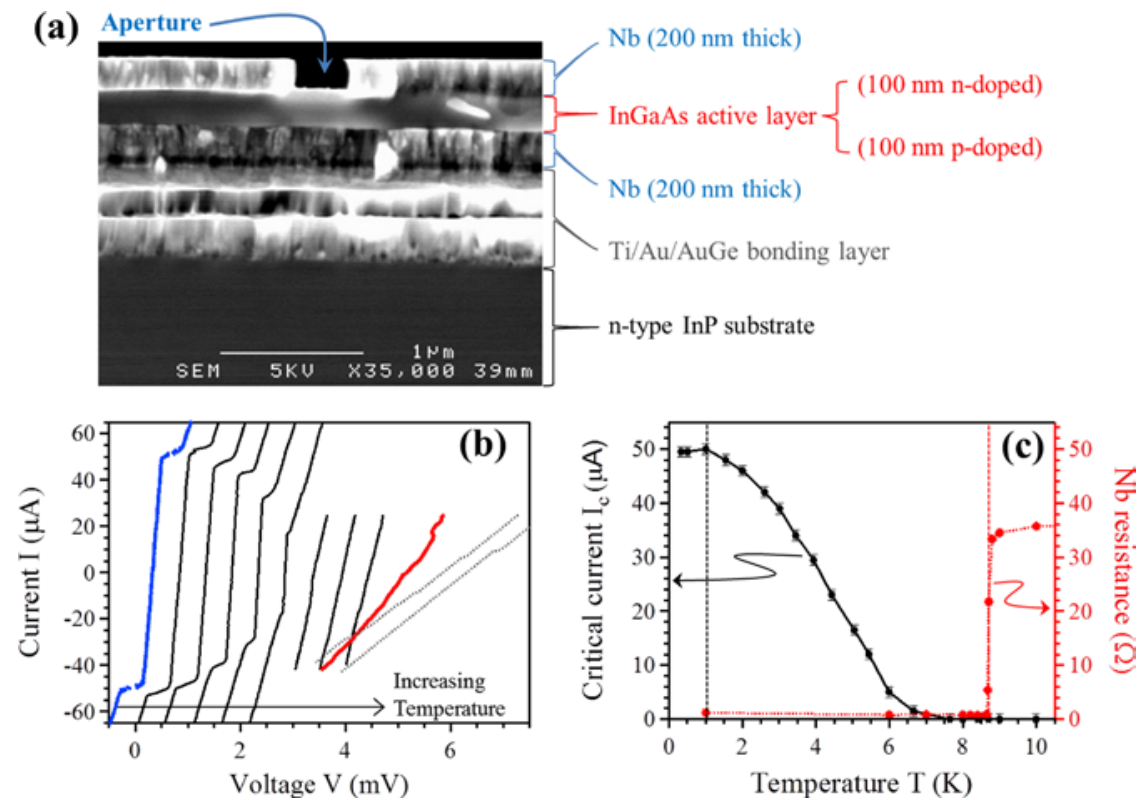

Figure 8: (a) Cross-sectional SEM of a $350 \mathrm{~nm}$ wide aperture for a SC LED fabricated in a $200 \mathrm{~nm}$-thick Nb surface electrode by the one-step balanced etching method. (b) Properties of an $80 \mathrm{~nm}$ Nb-InGaAs-Nb Josephson junction as a function of temperature: I-V curves of the Junction measured at temperatures between 0.33 and $9.99 \mathrm{~K}(0.33,1.00,2.00$, 3.01, 3.93, 5.04, 5.99, 8.00, 8.65, $8.7\left(T_{c}\right), 9.00$ and $9.99 \mathrm{~K}$ from left to right with incremental voltage offsets of $0.5 \mathrm{mV}$ for clarity). (c) Dependence of the critical current $I_{c}$ (solid black line) and the $\mathrm{Nb}$ resistance (dotted red line) on the junction temperature $T$, where the dashed vertical lines highlight the $I_{c}$ saturation at $I / I_{c} \sim 0.11$ and $T_{c} \sim 8.7 \mathrm{~K}$.

The typical Josephson junction transport characteristics are shown in figure 8(b), where the current $I$ through the junction is measured as a function of the applied voltage $V$. The temperature has been varied between 0.33 and $9.99 \mathrm{~K}$ for the displayed data. The left-most (blue dotted) curve shows the $0.33 \mathrm{~K}$ data taken at a higher resolution and exhibits the typical DC Josephson junction properties accompanied with hysteresis behavior [21, 22]. The invariably present slope in the measured data, which corresponds to a residual ohmic resistance of about $3 \mathrm{Ohm}$, is due to a non-zero serial normal resistance in the sample contact and wiring. The thick (red) line displays the $I-V$ characteristics at the critical temperature $\left(T_{c}=8.7 \mathrm{~K}\right)$ and shows the transition to the normal conductance of the $\mathrm{Nb}$ metal. In figure 8(c) the extracted values of the critical current $I_{c}$ and of the $\mathrm{Nb}$ resistance are plotted versus the sample temperature $T$. The Nb resistance exhibits the typical steep crossover between the super- and normal-conducting regimes at $8.7 \mathrm{~K}$ which is close to the intrinsic $\mathrm{Nb}$ transition temperature of $9.3 \mathrm{~K}$ and indicates the high quality of the deposited $\mathrm{Nb}$ layers. The maximum value of $I_{c}$ saturates at around $50 \mu \mathrm{A}$, which is, to the best of the authors' knowledge, among the largest values reported so far. This is in contrast to the observation of Josephson junction properties at $30 \mathrm{mK}$ in a similar device with $L=150 \mathrm{~nm}$ where $I_{c}$ was limited to around $2 \mu \mathrm{A}$ [20]. This clear enhancement of $I_{c}$ is due to the transition from a dirty to a clean junction. With the normal state resistance of the junction $\left(R_{N}=130 \mathrm{Ohm}\right)$ a characteristic voltage, $I_{c} \times R_{N}$, of $6.5 \mathrm{mV}$ can be extracted which suggests a high interface mobility. However, the saturation of $I_{c}$ at around $I / I_{c}=0.11$ indicates a still not perfectly transmissive junction. 


\section{Conclusion}

Under low RF power and low chamber pressure (5 Pa, $100 \mathrm{~W}$ ) etching conditions of $80 \mathrm{~nm}$ thick $\mathrm{Nb}$ electrodes apertures could be accurately transferred and processed according to their design. For the $200 \mathrm{~nm}$ thick $\mathrm{Nb}$ electrodes we found that higher RF power (200 W) resulted in sidewall deposition mediated by a strong ion bombardment and that an increased chamber pressure (30 Pa) led to isotropic etching by chemical reactions involving the absorbed gas ions. We also pointed out that the sidewall deposition layers act as sacrificial layers against isotropic chemical etching. We found that the combination of these unique etching phenomena led to a remarkable decrease of the size variation and slit-edge roughness. A three step processes was established, including the design of high contrast resist patterning, sacrificial layer formation under high RF power, and achievement of precise slit-widths by isotropic etching time control. Precise slit-width control of $\mathrm{Nb}$ apertures could be successfully demonstrated down to sizes as small as $20 \mathrm{~nm}$. High critical temperatures $(8.7 \mathrm{~K})$ and critical currents $(50 \mu \mathrm{A})$ could be justified for a clean and short S-Sm-S Josephson junction made of an $80 \mathrm{~nm} \mathrm{Nb}$ slit. Furthermore, the SC LED samples fabricated using this process are expected to be very effective for light extraction as well as for a source of entangled photon pairs that originate from a radiative recombination of an electron Cooper pair with two normal holes.

\section{Acknowledgments}

The authors thank Dr. H. Kumano and Dr. M. Endoh for valuable comments and helpful discussions. We also thank Dr. S. Odashima for preparation of Nb deposited samples for valuable experimental attempts. This work was supported in part by the Grant-in-Aid for Scientific Research (A), No. 21246048 from the Ministry of Education, Science, Sports, and Culture, and by the Hokkaido Innovation through NanoTechnology Support (HINTS) for nanofabrication. C. H. acknowledges the Japan Society for the Promotion of Science (JSPS) for providing financial support in the form of a JSPS Research Fellowship for Young Scientists. 


\section{References}

[1] Kwiat P G, Mattle K, Weinfurter H, Zeilinger A, Sergienko A V and Shih Y 1995 Phys. Rev. Lett. 754337

[2] Suemune I et al 2006 Jpn. J. Appl. Phys. 459264

[3] Hayashi Y, Tanaka K, Akazaki T, Jo M, Kumano H and Suemune I 2008 phys. stat. sol. (c) 52816

[4] Hayashi Y, Tanaka K, Akazaki T, Jo M, Kumano H and Suemune I 2008 Appl. Phys. Exp. 1011701

[5] Suemune I, Akazaki T, Tanaka K, Jo M, Uesugi K, Endo M, Kumano H and Hanamura E 2008 Microelectron. J. 39344

[6] Il'in K, Rall D, Siegel M, Engel A, Schilling A, Semenov A and Huebers H -W 2010 Physica C 470953

[7] Martines J M and Ono R H 1990 Appl. Phy. Lett, 57629

[8] Harada T, Gamo K and Namba S 1981 Jpn. J. Appl. Phys., 20259

[9] Lam S K H and Tilbrook D L 2003 Appl. Phy. Lett., 821078

[10] Hu C -K, Mazzeo N, Wind S J, Pearson D J and Ketchen M B 1991 Thin Solid Films, 206151

[11] Foxe T T, Hunt B D, Rogers C, Kleinsasser A W and Buhrman R A 1981 J. Vac. Sci. Technol. 191394

[12] Sasserath J N and Vivalda J 1990 J. Vac. Sci. Technol. A, 83914

[13] Sasserath J N 1990 J. Appl. Phys., 685324

[14] Curtis B J and Mantle H 1993 J. Vac. Sci. Technol., A 112846

[15] Kato Y and Michikami O 1984 Jpn. J. Appl. Phys., 23564

[16] Farrell H H and Strongin M 1973 Sur. Sci., 3818

[17] Farrell H H, Isaacs H S and Strongin M 1973 Sur. Sci., 3831

[18] Daccá A, Gemme G, Mattera L and Parodi R 1998 App. Sur. Sci. 126219

[19] Akaike H, Watanabe T, Fujimaki A and Hayakawa H 1995 Jpn. J. Appl. Phys. 341515

[20] Inoue R, Takayanagi H, Akazaki K and Suemune I 2010 Supercond. Sci. Technol. 23034025

[21] Beenacker C W J 1997 Rev. Mod. Phys. 69731

[22] Golubov A A, Kupriyanov M Yu and Il'ichev E 2004 Rev. Mod. Phys. 76411 